TITLE:

\title{
A Min-Max Theorem for Transversal Submodular Functions and Its Implications
}

\section{$\operatorname{AUTHOR}(\mathrm{S}):$}

Fujishige, Satoru; Tanigawa, Shin-ichi

\section{CITATION:}

Fujishige, Satoru ...[et al]. A Min-Max Theorem for Transversal Submodular Functions and Its Implications. SIAM Journal on Discrete Mathematics 2014, 28(4): 1855-1875

\section{ISSUE DATE:}

2014-10-02

URL:

http://hdl.handle.net/2433/191193

RIGHT:

(C) 2014, Society for Industrial and Applied Mathematics 


\title{
A MIN-MAX THEOREM FOR TRANSVERSAL SUBMODULAR FUNCTIONS AND ITS IMPLICATIONS*
}

\author{
SATORU FUJISHIGE ${ }^{\dagger}$ AND SHIN-ICHI TANIGAWA ${ }^{\dagger}$
}

\begin{abstract}
Huber and Kolmogorov [Towards minimizing k-submodular functions, in Proceedings of ISCO 2012, Lecture Notes in Comput. Sci. 7422, Springer, Heidelberg, 2012, pp. 451-462] introduced a concept of $k$-submodular function as a generalization of ordinary submodular (set) functions and bisubmodular functions and obtained a min-max theorem for the minimization of $k$-submodular functions. Also Kuivinen [Discrete Optim., 8 (2011), pp. 459-477] considered submodular functions on (product lattices of) diamonds and showed a min-max theorem for the minimization of submodular functions on diamonds. In the present paper we consider a common generalization of $k$-submodular functions and submodular functions on diamonds, which we call a transversal submodular function (a t-submodular function, for short). We show a min-max theorem for the minimization of t-submodular functions in terms of a new norm composed of $\ell_{1}$ and $\ell_{\infty}$ norms. This reveals a relationship between the obtained min-max theorem and that for the minimization of ordinary submodular set functions due to Edmonds [Submodular functions, matroids, and certain polyhedra, in Proceedings of the Calgary International Conference on Combinatorial Structures and Their Applications, R. Guy, H. Hanani, N. Sauer, and J. Schönheim, eds., Gordon and Breach, New York, 1970, pp. 69-87]. We also show how our min-max theorem for t-submodular functions can be used to prove the min-max theorem for $k$-submodular functions by Huber and Kolmogorov and that for submodular functions on diamonds by Kuivinen. Moreover, we show a counterexample to a characterization, given by Huber and Kolmogorov [Towards minimizing $k$-submodular functions, in Proceedings of ISCO 2012, Lecture Notes in Comput. Sci. 7422, Springer, Heidelberg, 2012, pp. 451-462], of extreme points of the $k$-submodular polyhedron and make it a correct one by fixing a flaw therein.
\end{abstract}

Key words. transversal submodular functions, $k$-submodular functions, submodular functions on lattices, min-max relation

AMS subject classifications. 90C27, 52B40, 52B12

DOI. $10.1137 / 130936415$

1. Introduction. Huber and Kolmogorov [7] introduced the concept of a $k$ submodular function, which is a generalization of ordinary submodular (set) functions and bisubmodular functions (see, e.g., [3, 5, 6]). Motivated by [11], Huber and Kolmogorov introduced convex polyhedra, what they call $k$-submodular polyhedra, associated with $k$-submodular functions. Earlier than Huber and Kolmogorov [7], Bouchet [2] also considered a class of $k$-submodular functions to define multimatroids as a generalization of delta-matroids [1,3]. Kolmogorov [8] also considered a concept of tree-submodularity, which is more general than $k$-submodularity. It was shown in [8] that polynomial solvability of the $k$-submodular function minimization implies that of the tree-submodular function minimization for all trees.

Huber and Kolmogorov [7] presented a min-max theorem that characterizes the minimum of a $k$-submodular function in terms of $\ell_{1}$ norm. Also Kuivinen [11] considered submodular functions on (product lattices of) diamonds and showed a min-max theorem for the minimization of submodular functions on diamonds.

Thapper and Živný [13] showed a dichotomy theorem that classifies the polynomialtime solvability of the minimization problems of explicitly given functions on finite

${ }^{*}$ Received by the editors September 10, 2013; accepted for publication (in revised form) June 9, 2014; published electronically October 23, 2014. This work was supported by JSPS Grant-in-Aid for Scientific Research (B) 25280004.

http://www.siam.org/journals/sidma/28-4/93641.html

${ }^{\dagger}$ Research Institute for Mathematical Sciences, Kyoto University, Kyoto 606-8502, Japan (fujishig@kurims.kyoto-u.ac.jp, tanigawa@kurims.kyoto-u.ac.jp). 


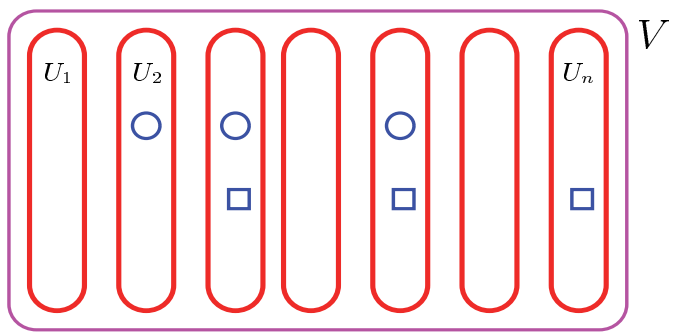

FIG. 1. A partition $\mathcal{U}$ of $V$ and subtransversals $T_{1}$ (of circles) and $T_{2}$ (of squares).

domains in terms of binary fractional polymorphisms (see $[12,13,14]$ for details). One of the important applications of this result is the tractability of the $k$-submodular function minimization problem and the minimization problem of submodular functions on lattices in the valued CSP model since its complexity was not known before. It, however, remains an open problem whether those functions can be minimized in polynomial time in the value oracle model.

In the present paper we consider a common generalization of $k$-submodular functions and submodular functions on diamonds, which we call a transversal submodular function (a $t$-submodular function, for short). We show a min-max theorem for the minimization of t-submodular functions in terms of a new norm composed of $\ell_{1}$ and $\ell_{\infty}$ norms. This reveals a relationship between the obtained min-max theorem and that for the minimization of ordinary submodular set functions due to Edmonds [4]. We also show how our min-max theorem for t-submodular functions can be used to prove the min-max theorem for $k$-submodular functions by Huber and Kolmogorov [7] and that for submodular functions on diamonds by Kuivinen [11]. Moreover, we show a counterexample to a characterization, given by Huber and Kolmogorov [7], of extreme points of the $k$-submodular polyhedron and make it a correct one by fixing a flaw therein.

The present paper is organized as follows. In section 2 we introduce the concept of transversal submodular (t-submodular) function and show a min-max theorem that characterizes the minimum of a t-submodular function in terms of a new norm composed of $\ell_{1}$ and $\ell_{\infty}$ norms. As special cases of t-submodular functions, we consider $k$-submodular functions in section 3 and submodular functions on lattices and, in particular, diamonds in section 4 in detail.

2. A min-max theorem for transversal submodular functions. Let $V$ be a nonempty finite set and $\mathcal{U} \equiv\left\{U_{1}, U_{2}, \ldots, U_{n}\right\}$ be a partition of $V$. A subset $T \subseteq V$ is called a transversal of $\mathcal{U}$ if $|T \cap U|=1$ for all $U \in \mathcal{U}$ and a subset of a transversal is called a subtransversal (or partial transversal). That is, a subset $T \subseteq V$ is called a subtransversal of $\mathcal{U}$ if $|T \cap U| \leq 1$ for all $U \in \mathcal{U}$. Denote by $\mathcal{T}$ the set of all subtransversals of $\mathcal{U}$. (See Figure 1.) Define for any $T \in \mathcal{T}$

$$
\mathcal{U}(T)=\{U \in \mathcal{U} \mid U \cap T \neq \emptyset\}
$$

For any $v \in V$ define $U(v)$ to be the unique $U \in \mathcal{U}$ that contains $v$. Note that $\mathcal{U}(T)=\{U(v) \mid v \in T\}$ for $T \in \mathcal{T}$.

We consider two binary operations $\nabla$ and $\triangle$ on $\mathcal{T}$ satisfying the condition that 
for all $T_{1}, T_{2} \in \mathcal{T}$

$$
\begin{array}{ll}
T_{1} \nabla T_{2} \in \mathcal{T}, \quad & \mathcal{U}\left(T_{1} \nabla T_{2}\right) \subseteq \mathcal{U}\left(T_{1}\right) \cup \mathcal{U}\left(T_{2}\right), \\
T_{1} \triangle T_{2} \in \mathcal{T}, & \mathcal{U}\left(T_{1} \triangle T_{2}\right) \subseteq \mathcal{U}\left(T_{1}\right) \cap \mathcal{U}\left(T_{2}\right) .
\end{array}
$$

Define a function $f: \mathcal{T} \rightarrow \mathbb{R}$ with $f(\emptyset)=0$ satisfying

$$
f\left(T_{1}\right)+f\left(T_{2}\right) \geq f\left(T_{1} \nabla T_{2}\right)+f\left(T_{1} \triangle T_{2}\right) \quad\left(\forall T_{1}, T_{2} \in \mathcal{T}\right) .
$$

We call $f$ a t-submodular function.

For any $x \in \mathbb{R}^{V}$ define

$$
\|x\|_{1, \infty}=\sum_{i=1}^{n} \max _{u \in U_{i}}|x(u)|
$$

This defines a norm on $\mathbb{R}^{V}$, which is a composition of $\ell_{1}$ and $\ell_{\infty}$ norms. Our main result is a min-max theorem based on the new norm $\|\cdot\|_{1, \infty}$ on $\mathbb{R}^{V}$.

Our general framework reveals a relationship between the general min-max relation for t-submodular functions and that for ordinary submodular set functions due to Edmonds [4].

Define a function $F: 2^{\mathcal{U}} \rightarrow \mathbb{R}$ as follows:

$$
F(\mathcal{X})=\min \{f(T) \mid T \in \mathcal{T}, \mathcal{U}(T) \subseteq \mathcal{X}\} \quad(\forall \mathcal{X} \subseteq \mathcal{U})
$$

Lemma 2.1. $F: 2^{\mathcal{U}} \rightarrow \mathbb{R}$ is a submodular function with $F(\emptyset)=0$.

Proof. For any $\mathcal{X}, \mathcal{Y} \subseteq \mathcal{U}$ there exist $T_{\mathcal{X}}, T_{\mathcal{Y}} \in \mathcal{T}$ such that

$$
\mathcal{U}\left(T_{\mathcal{X}}\right) \subseteq \mathcal{X}, \quad \mathcal{U}\left(T_{\mathcal{Y}}\right) \subseteq \mathcal{Y}, \quad F(\mathcal{X})=f\left(T_{\mathcal{X}}\right), \quad F(\mathcal{Y})=f\left(T_{\mathcal{Y}}\right)
$$

Hence, from (2.2)-(2.7) we have

$F(\mathcal{X})+F(\mathcal{Y})=f\left(T_{\mathcal{X}}\right)+f\left(T_{\mathcal{Y}}\right) \geq f\left(T_{\mathcal{X}} \nabla T_{\mathcal{Y}}\right)+f\left(T_{\mathcal{X}} \triangle T_{\mathcal{Y}}\right) \geq F(\mathcal{X} \cup \mathcal{Y})+F(\mathcal{X} \cap \mathcal{Y})$

We also have $F(\emptyset)=f(\emptyset)=0$.

Since $F: 2^{\mathcal{U}} \rightarrow \mathbb{R}$ is an ordinary submodular set function with $F(\emptyset)=0$, we have the submodular polyhedron associated with submodular function $F[6]$ defined by

$$
\mathrm{P}(F)=\left\{x \in \mathbb{R}^{\mathcal{U}} \mid \forall \mathcal{X} \subseteq \mathcal{U}: x(\mathcal{X}) \leq F(\mathcal{X})\right\}
$$

where $x(\mathcal{X})=\sum_{U \in \mathcal{X}} x(U)$ for all $\mathcal{X} \subseteq \mathcal{U}$. Moreover, we have Edmonds' min-max theorem for submodular function minimization [4] as follows (also see [6, Corollary 3.5]).

Proposition 2.2. For a submodular function $F: 2^{\mathcal{U}} \rightarrow \mathbb{R}$ with $F(\emptyset)=0$, we have

$$
\min \{F(\mathcal{X}) \mid \mathcal{X} \subseteq \mathcal{U}\}=\max \{x(\mathcal{U}) \mid x \leq \mathbf{0}, x \in \mathrm{P}(F)\}
$$

Moreover, if $F$ is integer-valued, there exists an integral maximizer of the right-hand side of (2.9).

Now we can easily see that

$$
\min \{f(T) \mid T \in \mathcal{T}\}=\min \{F(\mathcal{X}) \mid \mathcal{X} \subseteq \mathcal{U}\},
$$


which is equal to $F(\mathcal{U})$ since $F$ is monotone nonincreasing. Hence, we have the following lemma.

LEMMA 2.3.

$$
\min \{f(T) \mid T \in \mathcal{T}\}=\max \{x(\mathcal{U}) \mid x \leq \mathbf{0}, x \in \mathrm{P}(F)\} .
$$

Proof. The proof follows from (2.9) and (2.10).

It should be noted that since $F$ is monotone nonincreasing, every $x \in \mathrm{P}(F)$ is nonpositive, so that we may suppress the condition $x \leq \mathbf{0}$ appearing in (2.11).

For any $x \in \mathbb{R}^{\mathcal{U}}$ define $z_{x} \in \mathbb{R}^{V}$ by

$$
z_{x}(v)=x(U(v)) \quad(\forall v \in V) .
$$

Here it should be noted that $x(U(v))$ is the value of $x \in \mathbb{R}^{\mathcal{U}}$ for the coordinate $U(v) \in$ $\mathcal{U}$. We call such a vector $z_{x} \in \mathbb{R}^{V}$ a $\mathcal{U}$-componentwise constant vector associated with $x \in \mathbb{R}^{\mathcal{U}}$.

Lemma 2.4. Suppose we are given a nonpositive $x \in \mathbb{R}^{\mathcal{U}}$, i.e., $x \leq \mathbf{0}$. Then, we have $x \in \mathrm{P}(F)$ if and only if $z_{x} \in \mathrm{P}(f)$, where

$$
\mathrm{P}(f)=\left\{z \in \mathbb{R}^{V} \mid \forall T \in \mathcal{T}: z(T)\left(\equiv \sum_{v \in T} z(v)\right) \leq f(T)\right\} .
$$

Proof. Suppose $x \in \mathrm{P}(F)$. Then, for any $T \in \mathcal{T}$

$$
z_{x}(T)=x(\mathcal{U}(T)) \leq F(\mathcal{U}(T)) \leq f(T) .
$$

Hence, $z_{x} \in \mathrm{P}(f)$. Conversely, suppose $z_{x} \in \mathrm{P}(f)$ for $x \in \mathbb{R}^{\mathcal{U}}$ with $x \leq \mathbf{0}$. Then, for any $\mathcal{X} \subseteq \mathcal{U}$ and any $T \in \mathcal{T}$ such that $\mathcal{U}(T) \subseteq \mathcal{X}$ we have

$$
x(\mathcal{X}) \leq x(\mathcal{U}(T))=z_{x}(T) \leq f(T),
$$

where the first inequality holds since $x \leq \mathbf{0}$. This implies

$$
x(\mathcal{X}) \leq \min \{f(T) \mid T \in \mathcal{T}, \mathcal{U}(T) \subseteq \mathcal{X}\}=F(\mathcal{X}) .
$$

Hence, $x \in \mathrm{P}(F)$.

We are now ready to show the following theorem.

THEOREM 2.5. For any t-submodular function $f$ with $f(\emptyset)=0$ we have the following min-max relation:

$$
\min \{f(T) \mid T \in \mathcal{T}\}=\max \left\{-\|z\|_{1, \infty} \mid z \in \mathrm{P}(f)\right\} .
$$

Moreover, if $f$ is integer-valued, there exists an integral vector $z$ that attains the maximum on the right-hand side of (2.17).

Proof. Denote the right-hand side of (2.17) by RHS. It follows from Lemmas 2.3 and 2.4 that

$$
\begin{aligned}
\mathrm{RHS} & =\max \left\{-\|z\|_{1, \infty} \mid z \leq \mathbf{0}, \quad z \in \mathrm{P}(f)\right\} \\
& =\max \left\{-\left\|z_{x}\right\|_{1, \infty} \mid x \in \mathbb{R}^{\mathcal{U}}, x \leq \mathbf{0}, z_{x} \in \mathrm{P}(f)\right\} \\
& =\max \{x(\mathcal{U}) \mid x \leq \mathbf{0}, x \in \mathrm{P}(F)\} \\
& =\min \{F(\mathcal{X}) \mid \mathcal{X} \subseteq \mathcal{U}\} \\
& =\min \{f(T) \mid T \in \mathcal{T}\},
\end{aligned}
$$


where the first and second equalities are due to the hereditary property of polyhedron $\mathrm{P}(f)$ and the definition of norm $\|\cdot\|_{1, \infty}$.

Moreover, if $f$ is integer-valued, then so is the corresponding submodular function $F: 2^{\mathcal{U}} \rightarrow \mathbb{R}$. Therefore, there exists an integral $x \in \mathbb{R}^{\mathcal{U}}$ that attains the maximum of the right-hand side of (2.11) due to Proposition 2.2. Then $z_{x} \in \mathbb{R}^{V}$ defined by (2.12) is an integral maximizer of the right-hand side of (2.17), due to Lemmas 2.3 and 2.4 .

It should be noted that the proof of Theorem 2.5 shows that the maximum on the right-hand side of (2.17) is attained by a nonpositive $\mathcal{U}$-componentwise constant vector $z_{x} \in \mathbb{R}^{V}$ defined by (2.12), i.e., the maximizer $z_{x}$ takes on the same nonpositive value on each $U \in \mathcal{U}$. Because of this property the general min-max relation, Theorem 2.5, implies the min-max relations shown in [7] and [11]. We will give detailed arguments in what follows.

We consider $k$-submodular functions in section 3 and submodular functions on lattices and, in particular, diamonds in section 4 as special cases of t-submodular functions.

3. $k$-submodular functions. As an example of t-submodular functions we consider $k$-submodular functions due to Huber and Kolmogorov [7] and give a constructive proof of a min-max theorem. We also consider a characterization of extreme points of $k$-submodular polyhedra in the sense of Huber and Kolmogorov.

Let $V, \mathcal{U}$, and $\mathcal{T}$ be those appearing in section 2 .

3.1. Min-max theorems. For any $T, T^{\prime} \in \mathcal{T}$ define binary operations $\sqcup$ and $\sqcap$ on $\mathcal{T}$ by

$$
T \sqcup T^{\prime}=\left(T \cup T^{\prime}\right) \backslash \bigcup\left\{U \in \mathcal{U}|| U \cap\left(T \cup T^{\prime}\right) \mid=2\right\}, \quad T \sqcap T^{\prime}=T \cap T^{\prime} .
$$

Let $k=\max \{|U| \mid U \in \mathcal{U}\}$. A function $f: \mathcal{T} \rightarrow \mathbb{R}$ is called $k$-submodular if

$$
f(T)+f\left(T^{\prime}\right) \geq f\left(T \sqcup T^{\prime}\right)+f\left(T \sqcap T^{\prime}\right) \quad\left(\forall T, T^{\prime} \in \mathcal{T}\right) .
$$

This definition of a $k$-submodular function is equivalent to that given in [7] except that $|U|=k$ for all $U \in \mathcal{U}$ there. We assume $f(\emptyset)=0$. We can easily see that $k$-submodular functions are t-submodular functions with binary operations $\sqcup$ and $\sqcap$.

We call $(\mathcal{U}, f)$ a $k$-submodular system on $V$. Define a polyhedron

$$
\mathrm{P}(f)=\left\{x \in \mathbb{R}^{V} \mid \forall T \in \mathcal{T}: x(T) \leq f(T)\right\} .
$$

We call $\mathrm{P}(f)$ the $k$-submodular polyhedron associated with the $k$-submodular system $(\mathcal{U}, f)$.

Bouchet [2] considered $k$-submodular functions that were monotone nondecreasing and had the unit-increase property to define a set system called a multimatroid, a generalization of delta-matroids [1]. General $k$-submodular functions were considered by Huber and Kolmogorov [7]. They defined a polyhedron in a way slightly different from our $\mathrm{P}(f)$ in (3.3) by adding the following inequalities to those in (3.3):

$$
\forall U \in \mathcal{U}, \quad \forall X \in\left(\begin{array}{c}
U \\
2
\end{array}\right): x(X) \leq 0,
$$

where $\left(\begin{array}{c}U \\ 2\end{array}\right)$ is the set of all two-element subsets of $U$. We denote the " $k$-submodular polyhedron" in the sense of Huber and Kolmogorov by $\mathrm{P}_{2}(f)$, i.e.,

$$
\mathrm{P}_{2}(f)=\left\{x \in \mathbb{R}^{V} \mid \forall T \in \mathcal{T}: x(T) \leq f(T), \forall U \in \mathcal{U}, \forall X \in\left(\begin{array}{c}
U \\
2
\end{array}\right): x(X) \leq 0\right\} .
$$


Note that we have

$$
\mathrm{P}(f) \cap \mathbb{R}_{\leq 0}^{V}=\mathrm{P}_{2}(f) \cap \mathbb{R}_{\leq 0}^{V} \subseteq \mathrm{P}_{2}(f) \subseteq \mathrm{P}(f),
$$

where $\mathbb{R}_{<0}^{V}$ is the set of all nonpositive vectors in $\mathbb{R}^{V}$.

As a corollary of Theorem 2.5 we get the following corollary.

Corollary 3.1. For any $k$-submodular function $f: \mathcal{T} \rightarrow \mathbb{R}$ with $f(\emptyset)=0$,

$$
\min \{f(T) \mid T \in \mathcal{T}\}=\max \left\{-\|x\|_{1, \infty} \mid x \in \mathrm{P}(f)\right\} .
$$

Moreover, if $f$ is integer-valued, then there exists an integral $x$ that attains the maximum on the right-hand side of (3.7).

It should be noted that Corollary 3.1 also follows from the min-max relation for $k$-submodular functions shown by Huber and Kolmogorov [7].

For the polyhedron $\mathrm{P}_{2}(f)$ considered by Huber and Kolmogorov [7] we have the following theorem.

TheOREM 3.2. For any $k$-submodular function $f: \mathcal{T} \rightarrow \mathbb{R}$ with $f(\emptyset)=0$,

$$
\min \{f(T) \mid T \in \mathcal{T}\}=\max \left\{-\|x\|_{1, \infty} \mid x \in \mathrm{P}_{2}(f)\right\} .
$$

Moreover, if $f$ is integer-valued, then there exists an integral $x$ that attains the maximum on the right-hand side of (3.8).

Proof. By the proof of Theorem 2.5 there exists a nonpositive $\mathcal{U}$-componentwise constant maximizer $x^{*} \in \mathrm{P}(f)$ of (3.7), i.e., for each $U \in \mathcal{U}, x^{*}(v)=-\gamma_{U}$ for some $\gamma_{U} \geq 0$ for all $v \in U$. Since $\mathrm{P}_{2}(f) \subseteq \mathrm{P}(f)$ and $x^{*}$ also belongs to $\mathrm{P}_{2}(f)$, the maximum value in (3.8) is equal to that in (3.7). Similarly, the latter integrality property follows.

3.2. Constructive proof of Corollary 3.1. In the following we give another constructive proof of Corollary 3.1, which reveals fundamental properties of the $k$ submodular polyhedra and is interesting in its own right.

For any $x \in \mathrm{P}(f)$ (or $\left.x \in \mathrm{P}_{2}(f)\right)$ and $T \in \mathcal{T}$ we say $T$ is $x$-tight if $x(T)=f(T)$. We can easily show the following lemma (see [7]).

Lemma 3.3. For any $x \in \mathrm{P}_{2}(f)$ and $X, Y \in \mathcal{T}$, if $X$ and $Y$ are $x$-tight, then $X \sqcup Y$ and $X \sqcap Y$ are also $x$-tight.

It should be noted that the collection of vectors $x \in \mathrm{P}(f)$ with $x \leq \mathbf{0}$ plays an important rôle in our arguments and that such vectors belong to $\mathrm{P}_{2}(f)$.

For any $u \in V$ and $x \in \mathrm{P}(f)$ define

$$
\hat{\mathrm{c}}(x, u)=\max \left\{\alpha \in \mathbb{R} \mid x+\alpha \chi_{u} \in \mathrm{P}(f)\right\},
$$

where $\chi_{u}$ is the unit vector in $\mathbb{R}^{V}$ with $\chi_{u}(u)=1$ and $\chi_{u}(v)=0$ for all $v \in V \backslash\{u\}$. Note that $\hat{\mathrm{c}}(x, u)$ can be expressed as

$$
\hat{\mathrm{c}}(x, u)=\min \{f(X)-x(X) \mid u \in X \in \mathcal{T}\} .
$$

We call $\hat{\mathrm{c}}(x, u)$ the saturation capacity associated with $x$ and $u$. If $\hat{\mathrm{c}}(x, u)=0$, we call $u$ saturated, and otherwise $(\hat{\mathrm{c}}(x, u)>0)$, nonsaturated. Define $\operatorname{sat}(x)$ to be the set of saturated elements associated with $x$. We see that $u$ is saturated if and only if there exists at least one $x$-tight set $X$ such that $u \in X$. Let us denote by $\mathcal{T}(x)$ the collection of $x$-tight sets.

For any $x \in \mathrm{P}(f)$ and any saturated $u \in V$ define the dependence function

$$
\operatorname{dep}(x, u)=\left\{v \in V \mid \exists \beta>0: x+\beta\left(\chi_{u}-\chi_{v}\right) \in \mathrm{P}(f)\right\} .
$$


This can be rewritten as

$$
\operatorname{dep}(x, u)=\bigcap\{X \mid u \in X \in \mathcal{T}(x)\} .
$$

Here, it should be noted that we have $\operatorname{dep}(x, u) \in \mathcal{T}(x)$ if $x \in \mathrm{P}_{2}(f)$ (due to Lemma $3.3)$ but not necessarily otherwise. If $x \in \mathrm{P}_{2}(f)$, then $\operatorname{dep}(x, u)$ is the unique minimal $x$-tight set containing $u$ since $\mathcal{T}(x)$ for $x \in \mathrm{P}_{2}(f)$ is closed with respect to set intersection $\cap(=\sqcap)$ due to Lemma 3.3.

Furthermore, for any $u \in \operatorname{sat}(x)$ and $v \in \operatorname{dep}(x, u) \backslash\{u\}$ define

$$
\tilde{\mathrm{c}}(x, u, v)=\max \left\{\beta \in \mathbb{R} \mid x+\beta\left(\chi_{u}-\chi_{v}\right) \in \mathrm{P}(f)\right\}>0,
$$

which is called the exchange capacity for $u$ and $v \in \operatorname{dep}(x, u) \backslash\{u\}$ associated with $x$. This can also be rewritten as

$$
\tilde{\mathrm{c}}(x, u, v)=\min \{f(X)-x(X) \mid X \in \mathcal{T}, u \in X, v \notin X\} .
$$

The concepts of sat, $\hat{c}$, dep, and $\tilde{c}$ generalize those defined for ordinary submodular polyhedra (see $[6]$ ).

For any nonempty $W \subseteq V$ and $x \in \mathbb{R}^{V}$ we define $x^{W} \in \mathbb{R}^{W}$ by $x^{W}(v)=x(v)$ for all $v \in W$. Also define $\left(\mathcal{U}^{W}, f^{W}\right)$ to be the restriction of the $k$-submodular system $(\mathcal{U}, f)$ on $V$ to $W$ as follows. Let $\mathcal{U}^{W}=\{U \cap W \mid U \in \mathcal{U}, U \cap W \neq \emptyset\}, \mathcal{T}^{W}=$ $\{T \cap W \mid T \in \mathcal{T}\}$, and $f^{W}(T)=f(T)$ for all $T \in \mathcal{T}^{W}$. For $k^{\prime}=\max \left\{|U| \mid U \in \mathcal{U}^{W}\right\}$, $\left(\mathcal{U}^{W}, f^{W}\right)$ is a $k^{\prime}$-submodular system on $W$. For any nonempty $T \in \mathcal{T}, f^{T}$ is an ordinary submodular function on $2^{T}$, which defines the associated base polyhedron

$$
\mathrm{B}\left(f^{T}\right)=\left\{x \in \mathbb{R}^{T} \mid \forall X \subset T: x(X) \leq f(X), x(T)=f(T)\right\} .
$$

(See $[6]$.

In order to prove Corollary 3.1 we will show some lemmas.

Lemma 3.4. For any $x \in \mathrm{P}(f)$ and $T \in \mathcal{T}$ we have

$$
f(T) \geq x(T) \geq-\|x\|_{1, \infty} .
$$

Proof. The proof easily follows from the definitions of $\mathrm{P}(f)$ and $\|x\|_{1, \infty}$.

Let $x^{*}$ be a maximizer of the right-hand side of (3.7). Because of the definition of $\mathrm{P}(f)$ we can assume that $x^{*} \leq \mathbf{0}$. Recall that $u \in V$ is saturated if for every $\alpha>0$ we have $x^{*}+\alpha \chi_{u} \notin \mathrm{P}(f)$, and nonsaturated otherwise. If $x^{*}(u)<0$ for some nonsaturated $u$, then we can make $u$ saturated or $x^{*}(u)=0$ without increasing the norm $\left\|x^{*}\right\|_{1, \infty}$. Hence, we further assume that $u$ is saturated for every $u \in V$ with $x^{*}(u)<0$.

We fix such a maximizer $x^{*}$ in the following argument.

Recall that $\mathcal{T}\left(x^{*}\right)$ is the collection of $x^{*}$-tight sets. It is a crucial fact that since $x^{*} \leq \mathbf{0}, \mathcal{T}\left(x^{*}\right)$ is closed with respect to binary operations $\sqcup$ and $\sqcap$, due to (3.6) and Lemma 3.3.

Lemma 3.5. For every $u \in V$ with $x^{*}(u)<0$ we have $\operatorname{dep}\left(x^{*}, u\right) \in \mathcal{T}\left(x^{*}\right)$.

Proof. By the assumption, $u$ is saturated and $x^{*} \leq \mathbf{0}$. It follows from Lemma 3.3 that $\operatorname{dep}\left(x^{*}, u\right) \in \mathcal{T}\left(x^{*}\right)$.

We write $\operatorname{dep}\left(x^{*}, u\right)$ as $D(u)$ for simplicity in what follows. Recall that for any $v \in V U(v)$ is the unique set $U \in \mathcal{U}$ such that $v \in U$.

Lemma 3.6. Suppose that $u \in V$ and $x^{*}(u)<0$. Then for $v \in V$ with $D(u) \cap$ $U(v)=\emptyset$ we have $x^{*}(v)=0$ or

$$
\left|(D(u) \cup D(v)) \cap U_{i}\right| \neq 2 \quad(\forall i=1, \ldots, n) .
$$


Proof. If $x^{*}(v)<0$ and some $U_{i}$ violates (3.17), then $v \in(D(u) \sqcup D(v)) \sqcap D(v) \subset$ $D(v)$, which contradicts the minimality of $D(v)$.

Let $u$ be an element of $V$ such that $x^{*}(u)<0$. Then, if for every $w \in D(u)$ we have $x^{*}(w)=\min \left\{x^{*}(v) \mid v \in U(w)\right\}$, we call u legitimate. Also, if for some $w \in D(u)$ we have $x^{*}(w)>\min \left\{x^{*}(v) \mid v \in U(w)\right\}$, we say $u$ is not legitimate with $w$.

The following is a key lemma.

Lemma 3.7. For any $U \in \mathcal{U}$ with $\min \left\{x^{*}(v) \mid v \in U\right\}<0$ let $W$ be the set of all the minimizers of $\min \left\{x^{*}(v) \mid v \in U\right\}$. Then there exists a legitimate $w \in W$.

Proof. Suppose, on the contrary, that no element in $W$ is legitimate. Then, $|D(w)|>1$ for all $w \in W$. For each $w \in W$ let $w^{-}$be an element of $D(w) \backslash\{w\}$ such that $x^{*}\left(w^{-}\right)>\min \left\{x^{*}(v) \mid v \in U\left(w^{-}\right)\right\}$. Put $z_{w^{-}}=x^{*}\left(w^{-}\right)-\min \left\{x^{*}(v) \mid v \in\right.$ $\left.U\left(w^{-}\right)\right\}$.

Now, for each $w \in W$ there exists some (sufficiently small) $\alpha_{w}>0$ such that $y_{w} \equiv x^{*}+\alpha_{w}\left(\chi_{w}-\chi_{w^{-}}\right) \in \mathrm{P}(f)$ and $\alpha_{w} \leq \min \left\{z_{w^{-}},-x^{*}(w)\right\}$. It follows that a convex combination $y^{*}$ of $y_{w}(w \in W)$ with positive coefficients has a norm $\left\|y^{*}\right\|_{1, \infty}$ smaller than $\left\|x^{*}\right\|_{1, \infty}$, a contradiction.

Now, for given $x^{*}$, we find a minimizer $T \in \mathcal{T}$ of $f$ by the following procedure.

Procedure Find_Min

Step 1: $\tilde{\mathcal{U}} \leftarrow\left\{U \in \mathcal{U} \mid \exists u \in U: x^{*}(u)<0\right\}$, $T \leftarrow \emptyset$

Step 2: While $\tilde{\mathcal{U}} \neq \emptyset$, do the following:

(1) Choose $U \in \tilde{\mathcal{U}}$ and let $\hat{u}$ be a legitimate element of $U$.

(2) $T \leftarrow T \cup D(\hat{u})$, $\tilde{\mathcal{U}} \leftarrow \tilde{\mathcal{U}} \backslash\{U(v) \mid v \in D(\hat{u})\}$.

Step 3: Return $T$.

The following lemma completes the proof of the min-max relation in Corollary 3.1. Lemma 3.8. Procedure Find_Min finds $T \in \mathcal{T}$ such that $-\left\|x^{*}\right\|_{1, \infty}=f(T)$.

Proof. It follows from Lemma 3.7 that we can find a legitimate $\hat{u}$ in Step 2. Furthermore, Lemma 3.6 validates $T \in \mathcal{T}$ with $T$ being $x^{*}$-tight. The finally obtained $T$ satisfies that $T \cap U \neq \emptyset$ for all $U \in \mathcal{U}$ with $\min \left\{x^{*}(v) \mid v \in U\right\}<0$ and that for all $u \in T$ we have $x^{*}(u)=\min \left\{x^{*}(v) \mid v \in U(u)\right\}$. Hence, $-\left\|x^{*}\right\|_{1, \infty}=x^{*}(T)=$ $f(T)$.

Now we show the latter half of Corollary 3.1, the integrality property. Note that by definition $\mathrm{P}(f)$ is hereditary, i.e., closed downward, so that there exists an integral $x$ in $\mathrm{P}(f)$.

Consider the following procedure.

\section{Procedure Find_Max}

Step 0: Let $x$ be an integral nonpositive vector in $\mathrm{P}(f)$.

Step 1: While there exists a nonsaturated $v \in V$ with $x(v)<0$, do the following: $\alpha \leftarrow \min \{-x(v), \hat{\mathrm{c}}(x, v)\}$, $x \leftarrow x+\alpha \chi_{v}$.

Step 2: $\tilde{\mathcal{U}} \leftarrow\{U \in \mathcal{U} \mid \exists u \in U: x(u)<0\}$, $T \leftarrow \emptyset$. 
Step 3: While $\tilde{\mathcal{U}} \neq \emptyset$, do the following:

(1) Choose $U \in \tilde{\mathcal{U}}$.

(2) Define $W=\{u \in U \mid x(u)=\min \{x(v) \mid v \in U\}\}$.

(3) Choose $u \in W$.

(3-1) If $u$ is not legitimate with $w \in D(u) \backslash\{u\}$, then

(a) $\beta \leftarrow \min \{-x(u), \tilde{\mathrm{c}}(x, u, w), x(w)-\min \{x(v) \mid v \in U(w)\}\}$,

(b) $x \leftarrow x+\beta\left(\chi_{u}-\chi_{w}\right)$,

(c) If $\exists v \in U: x(v)<0$, then go to (2); else remove $U$ from $\tilde{\mathcal{U}}$.

(3-2) If $u$ is legitimate, then

$T \leftarrow T \cup D(u)$,

$\tilde{\mathcal{U}} \leftarrow \tilde{\mathcal{U}} \backslash\{U(v) \mid v \in D(u)\}$.

Step 4: Return $x$.

Lemma 3.9. Suppose $f$ is integer-valued. Starting with an integral $x \in \mathrm{P}(f)$ with $x \leq \mathbf{0}$, Procedure Find_Max finds an integral maximizer for the min-max relation in Corollary 3.1.

Proof. During the execution of Procedure Find_Max, $x$ remains integral. If $u$ in (3) of Step 3 is not legitimate, $x(u)$ becomes larger, and when $|W| \geq 2, W$ becomes smaller. Hence, repeating (2), (3), and (4) in Step 3, we find a legitimate $u$ or we get $x$ with $x(v)=0$ for all $v \in U$. It follows that Procedure Find_Max terminates after a finite number of iterations and the finally obtained integral $x$ and subtransversal $T$ give max and min solutions, similarly as in the proof of Lemma 3.8.

This completes a constructive proof of Corollary 3.1.

3.3. Extreme points of $\mathbf{P}_{2}(\boldsymbol{f})$. Huber and Kolmogorov [7] presented a characterization of extreme points of $\mathrm{P}_{2}(f)$ for a $k$-submodular function $f$. In particular, as a necessary condition, they state that if $x \in \mathbb{R}^{V}$ is a nonzero extreme point of $\mathrm{P}_{2}(f)$ then there is a nontrivial ${ }^{1}$ chain $\emptyset=T_{0} \subset T_{1} \subset \cdots \subset T_{k}$ of elements in $\mathcal{T}$ such that

(i) $\left|T_{i} \backslash T_{i-1}\right|=1$ for $1 \leq i \leq k$ and

(ii) $T_{i}$ is $x$-tight for $0 \leq i \leq k$.

We give a counterexample to this claim by showing the existence of a nonzero extreme point that does not satisfy (i).

Let $U_{1}=\left\{v_{1}, v_{2}, v_{3}\right\}$ and $U_{2}=\left\{u_{1}, u_{2}, u_{3}\right\}$. Let $V=U_{1} \cup U_{2}, \mathcal{U}=\left\{U_{1}, U_{2}\right\}$, and $M$ be any integer greater than 5 . Define $f: \mathcal{T} \rightarrow \mathbb{R}$ by

$$
\begin{aligned}
& f(\emptyset)=0, \\
& f\left(\left\{v_{1}\right\}\right)=-1, \quad f\left(\left\{u_{1}\right\}\right)=1, \\
& f\left(\left\{v_{i}\right\}\right)=f\left(\left\{u_{i}\right\}\right)=M \text { for } i=2,3, \\
& f\left(\left\{u_{1}, v_{1}\right\}\right)=-2, \\
& f\left(\left\{u_{i}, v_{j}\right\}\right)=f\left(\left\{u_{i}\right\}\right)+f\left(\left\{v_{j}\right\}\right) \text { for } i, j=1,2,3 \text { with }(i, j) \neq(1,1) .
\end{aligned}
$$

Lemma 3.10. The function $f$ defined above is $k$-submodular for $k=3$.

Proof. Take any $T, T^{\prime} \in \mathcal{T}$ and let us check $f(T)+f\left(T^{\prime}\right) \geq f\left(T \sqcup T^{\prime}\right)+f\left(T \sqcap T^{\prime}\right)$. We may assume $T \not \subset T^{\prime}$ and $T^{\prime} \not \subset T$. We shall use the fact that $f\left(\left\{u_{i}\right\}\right)+f\left(\left\{u_{j}\right\}\right) \geq 0$ and $f\left(\left\{v_{i}\right\}\right)+f\left(\left\{v_{j}\right\}\right) \geq 0$ for any distinct $i, j$.

1. If $|T|=1$ and $\left|T^{\prime}\right|=1$, denote $T=\{x\}$ and $T^{\prime}=\{y\}$.

\footnotetext{
${ }^{1}$ By a nontrivial chain, we mean $k \geq 1$.
} 
- If $U(x)=U(y)$, then $T \sqcup T^{\prime}=\emptyset$ and $T \sqcap T^{\prime}=\emptyset$. Thus $f(T)+f\left(T^{\prime}\right)=$ $f(\{x\})+f(\{y\}) \geq 0=f\left(T \sqcup T^{\prime}\right)+f\left(T \sqcap T^{\prime}\right)$.

- Otherwise, $T \sqcup T^{\prime}=\{x, y\}$ and $T \sqcap T^{\prime}=\emptyset$.

If $\{x, y\}=\left\{v_{1}, u_{1}\right\}$, then $f(T)+f\left(T^{\prime}\right)=0>-2=f\left(T \sqcup T^{\prime}\right)+f\left(T \sqcap T^{\prime}\right)$. If $\{x, y\} \neq\left\{v_{1}, u_{1}\right\}$, then $f(T)+f\left(T^{\prime}\right)=f(\{x\})+f(\{y\})=f\left(T \sqcup T^{\prime}\right)+$ $f\left(T \sqcap T^{\prime}\right)$.

2. If $|T|=2$ and $\left|T^{\prime}\right|=1$, denote $T=\{x, y\}$ and $T^{\prime}=\{z\}$. We may assume that $U(y)=U(z)$. Then $T \sqcup T^{\prime}=\{x\}$ and $T \sqcap T^{\prime}=\emptyset$. Hence,

- If $\{x, y\}=\left\{v_{1}, u_{1}\right\}$, then $f(T)+f\left(T^{\prime}\right)=-2+M \geq \max \left\{f\left(\left\{v_{1}\right\}\right), f\left(\left\{u_{1}\right\}\right)\right\}$ $\geq f(\{x\})=f\left(T \sqcup T^{\prime}\right)+f\left(T \sqcap T^{\prime}\right)$.

- Otherwise, $f(T)+f\left(T^{\prime}\right)=f(\{x\})+f(\{y\})+f(\{z\}) \geq f(\{x\})=$ $f\left(T \sqcup T^{\prime}\right)+f\left(T \sqcap T^{\prime}\right)$.

3. If $|T|=2$ and $\left|T^{\prime}\right|=2$, denote $T=\{x, y\}$ and $T^{\prime}=\{z, w\}$.

- If $\{x, y\}=\left\{v_{1}, u_{1}\right\}$, then $f\left(T \sqcup T^{\prime}\right) \leq 1$ and $f\left(T \sqcap T^{\prime}\right) \leq 1$. Therefore, $f(T)+f\left(T^{\prime}\right)=-2+f(\{z\})+f(\{w\}) \geq-3+M \geq f\left(T \sqcup T^{\prime}\right)+f\left(T \sqcap T^{\prime}\right)$.

- Otherwise, we may assume $\{z, w\} \neq\left\{v_{1}, u_{1}\right\}$. If $y=w$, then $T \sqcup T^{\prime}=$ $\{y\}$ and $T \sqcap T=\{y\}$, and hence $f(T)+f\left(T^{\prime}\right)=f(\{x\})+f(\{z\})+$ $2 f(\{y\}) \geq 2 f(\{y\})=f\left(T \sqcup T^{\prime}\right)+f\left(T \sqcap T^{\prime}\right)$. If $y \neq w$, we may assume $T \cap T^{\prime}=\emptyset$. Then $T \sqcup T^{\prime}=\emptyset$ and $T \sqcap T^{\prime}=\emptyset$, and hence $f(T)+f\left(T^{\prime}\right)=$ $f(\{x\})+f(\{y\})+f(\{z\})+f(\{w\}) \geq 0=f\left(T \sqcup T^{\prime}\right)+f\left(T \sqcap T^{\prime}\right)$.

Now consider the nonzero $x^{*} \in \mathbb{R}^{V}$ given by

$$
\begin{aligned}
& x^{*}\left(v_{1}\right)=-2, \quad x^{*}\left(v_{2}\right)=2, \quad x^{*}\left(v_{3}\right)=-2, \\
& x^{*}\left(u_{1}\right)=0, \quad x^{*}\left(u_{2}\right)=0, \quad x^{*}\left(u_{3}\right)=0 .
\end{aligned}
$$

We can see by exhaustive checking that $x^{*} \in \mathrm{P}_{2}(f)$ and the following equations hold:

$$
\begin{aligned}
& x^{*}\left(\left\{v_{1}, u_{1}\right\}\right)=f\left(\left\{v_{1}, u_{1}\right\}\right), \\
& x^{*}\left(\left\{v_{1}, v_{2}\right\}\right)=x^{*}\left(\left\{v_{2}, v_{3}\right\}\right)=0, \\
& x^{*}\left(\left\{u_{1}, u_{2}\right\}\right)=x^{*}\left(\left\{u_{2}, u_{3}\right\}\right)=x^{*}\left(\left\{u_{3}, u_{1}\right\}\right)=0 .
\end{aligned}
$$

Since the system of six equations in (3.18) uniquely determines the solution $x^{*}, x^{*}$ is an extreme point of $\mathrm{P}_{2}(f)$.

Note that for any chain of elements in $\mathcal{T}$ satisfying condition (i), condition (ii) is violated for $x=x^{*}$, since $x^{*}\left(v_{i}\right)<f\left(\left\{v_{i}\right\}\right)$ for any $v_{i}$ and $x^{*}\left(u_{i}\right)<f\left(\left\{u_{i}\right\}\right)$ for any $u_{i}$. Hence, $x^{*}$ cannot be any extreme point of $\mathrm{P}_{2}(f)$ that corresponds to the conditions given by Huber and Kolmogorov [7].

We have shown that the conditions provided in [7] do not give an exact characterization of extreme points of $\mathrm{P}_{2}(f)$. We will give a correct characterization of extreme points of $\mathrm{P}_{2}(f)$. Let $(\mathcal{U}, f)$ be a $k$-submodular system on $V$.

We first show some lemmas.

LEMma 3.11. For a nonempty $T \in \mathcal{T}$ let $x$ be a vector in $\mathbb{R}^{V}$ satisfying

(A) $x^{T} \in \mathrm{B}\left(f^{T}\right)$,

(B) For each $u \in T$,

(B1) if $x(u) \geq 0$, then $x(v)=-x(u)$ for all $v \in U(u) \backslash\{u\}$;

(B2) otherwise,

(1) $x(v)=x(u)$ for all $v \in U(u) \backslash\{u\}$ but one $v$ with $x(v)=-x(u)$ or

(2) $x(v)=0$ for all $v \in U(u) \backslash\{u\}$.

Then we have $x^{Z} \in \mathrm{P}_{2}\left(f^{Z}\right)$ for $Z=\bigcup\{U(u) \mid u \in T\}$. 
Proof. For any $X \in \mathcal{T}$ such that $X \subseteq Z$, we have

$$
\begin{aligned}
x(X) & =x(X)+x(T)-f(T) \\
& \leq x(X \sqcup T)+x(X \sqcap T)-f(T) \\
& \leq f(X \sqcup T)+f(X \sqcap T)-f(T) \\
& \leq f(X),
\end{aligned}
$$

where the first inequality follows from (B) since $x$ satisfies (3.4) and the second inequality follows from (A) and the fact that $X \sqcup T, X \sqcap T \subseteq T$. It follows from (3.19) and (B) that $x^{Z} \in \mathrm{P}_{2}\left(f^{Z}\right)$.

We also have the following lemma.

LEMMA 3.12. For a given $x \in \mathrm{P}_{2}(f)$ and a nonempty $T \in \mathcal{T}$ suppose that $x^{T} \in \mathrm{B}\left(f^{T}\right)$. Let $Z=\bigcup\{U(u) \mid u \in T\}$. For an element $u \in T$ define $y \in \mathbb{R}^{Z}$ by $y(v)=x(v)$ for all $v \in Z \backslash(U(u) \backslash\{u\})$ and $y(v)$ for all $v \in(U(u) \backslash\{u\})$ according to (B1) and (B2), replacing $x$ by $y$, in Lemma 3.11. Then we have $y \in \mathrm{P}_{2}\left(f^{Z}\right)$.

Proof. Since $x \in \mathrm{P}_{2}(f)$, similarly as in (3.19) we can show that $y \in \mathrm{P}_{2}\left(f^{Z}\right)$.

For $U \in \mathcal{U}$ consider the system of linear inequalities

$$
x(u)+x(v) \leq 0 \quad\left(\forall\{u, v\} \in\left(\begin{array}{c}
U \\
2
\end{array}\right)\right) .
$$

Denote by $C_{2}^{U}$ the cone of feasible solutions of (3.20). We call $\{u, v\}$ a tight pair for a feasible solution $x^{*}$ if the inequality of (3.20) for the pair $\{u, v\}$ holds with equality for $x=x^{*}$.

Lemma 3.13. Suppose $|U| \geq 3$. The cone $C_{2}^{U}$ is pointed and its extreme rays are given by $x(u)=\alpha$ and $x(v)=-\alpha$ for all $v \in U \backslash\{u\}$ with a parameter $\alpha \geq 0$ for all $u \in U$. Every componentwise maximal solution $x^{*}$ of (3.20) lies on an extreme ray of $C_{2}^{U}$, and if $x^{*} \neq \mathbf{0}$, the set of the tight pairs for $x^{*}$ forms a star with center $u$ such that $x^{*}(u)>0$.

Proof. Since $|U| \geq 3$, if we replace all the inequalities of (3.20) by equations, it gives the unique solution $x=\mathbf{0}$. Hence, $C_{2}^{U}$ is pointed. Moreover, for any componentwise maximal feasible solution $x^{*}$, if $x^{*} \neq \mathbf{0}$, there exists only one $u \in U$ such that $x^{*}(u)>0$. Since $x^{*}$ is componentwise maximal, we must have $x^{*}(v)=-x^{*}(u)$ for all $v \in U \backslash\{u\}$. Hence, $x^{*}$ lies on an extreme ray of $C_{2}^{U}$ and the tight pairs form a star with center $u$.

Note that every extreme vector (lying on an extreme ray) of $C_{2}^{U}$ is componentwise maximal.

For any subset $\mathcal{E} \subseteq\left(\begin{array}{c}U \\ 2\end{array}\right)$ we regard $\mathcal{E}$ as the edge set of an undirected graph $G=(U, \mathcal{E})$ with vertex set $U$.

Lemma 3.14. For any subset $\mathcal{E} \subseteq\left(\begin{array}{c}U \\ 2\end{array}\right)$ the system of equations

$$
x(u)+x(v)=0 \quad(\forall\{u, v\} \in \mathcal{E})
$$

uniquely determines the solution $x=\mathbf{0}$ if and only if every connected component of the graph $G=(U, \mathcal{E})$ contains at least one odd cycle.

Proof. Suppose that every connected component of the graph $G=(U, \mathcal{E})$ contains at least one odd cycle. Since equations in (3.21) for an odd cycle determine $x(v)=0$ for elements (vertices) $v$ on the cycle, (3.21) determines $x(v)=0$ for other elements $v$ in the same connected component.

Conversely, suppose that (3.21) determines the unique solution $x=\mathbf{0}$. Then we must have $\bigcup \mathcal{E}=U$. If some connected component having at least two vertices does 
not contain odd cycles, then it forms a bipartite graph. Hence, the values $x(v)$ for vertices $v$ in the connected component are not uniquely determined. (For, if $x\left(v_{0}\right)$ for a vertex $v_{0}$ of the bipartite graph is increased by $\alpha$, then increasing $x(v)$ for every $v$ at an even distance from $v_{0}$ by $\alpha$ and decreasing $x(v)$ for every $v$ at an odd distance from $v_{0}$ by $\alpha$ keep $x$ satisfying (3.21) for any $\alpha \in \mathbb{R}$.) Hence, every connected component has at least one odd cycle.

For $x^{T} \in \mathrm{B}\left(f^{T}\right)$ define a directed graph $G_{x}^{T}=\left(T, A_{x}\right)$ with the vertex set $T$ and the $\operatorname{arc}$ set $A_{x}$ given by

$$
A_{x}=\{(u, v) \mid u \in T, v \in \operatorname{dep}(x, u) \backslash\{u\}\} .
$$

Let $H_{x}^{i}=\left(S_{x}^{i}, B_{x}^{i}\right)(i \in I)$ be the strongly connected components of $G_{x}^{T}$. Choose any $w^{i} \in S_{x}^{i}$ for each $i \in I$. Then we call the set $W=\left\{w^{i} \mid i \in I\right\}$ a covering set of $G_{x}^{T}$.

It is known [6] that for any maximal chain of tight sets in $\mathcal{T}(x) \cap 2^{T}$

$$
\emptyset=T_{0} \subset T_{1} \subset \cdots \subset T_{p}=T
$$

the collection of the difference sets $T_{j} \backslash T_{j-1}(j=1, \ldots, p)$ is exactly the collection of vertex sets $S_{x}^{i}(i \in I)$ of the strongly connected components of $G_{x}^{T}$; in particular, $p=|I|$.

LEMma 3.15. For any $x \in \mathrm{P}_{2}(f)$ and nonempty $T \in \mathcal{T}$ suppose that the following three statements hold:

(1) For every tight set $T^{\prime} \in \mathcal{T}(x)$ we have $T^{\prime} \subseteq \bigcup\{U(u) \mid u \in T\}$.

(2) $x^{T} \in \mathrm{B}\left(f^{T}\right)$.

(3) (B) in Lemma 3.11 is satisfied.

If for some $i_{0} \in I$

(a) we have $\left|S_{x}^{i_{0}}\right| \geq 2$ and

(b) for some distinct $u, v \in S_{x}^{i_{0}}$ we have $x(u) \neq 0$ and $x(v) \neq 0$, and letting $\mathcal{E}_{u}$ and $\mathcal{E}_{v}$ be, respectively, the sets of all tight pairs for $U(u)$ and $U(v)$, the connected component of graph $\left(U(u), \mathcal{E}_{u}\right)$ containing $u$ and that of $\left(U(v), \mathcal{E}_{v}\right)$ containing $v$ are both bipartite (more specifically, stars),

then $x$ is not an extreme point of $\mathrm{P}_{2}(f)$.

Proof. Under the assumption of the present lemma let $u$ and $v$ be those appearing in (b). Define

$$
\begin{aligned}
& \alpha_{1}=\min \{|x(u)|,|x(v)|\} \\
& \alpha_{2}=\min \left\{f\left(T^{\prime}\right)-x\left(T^{\prime}\right)\left|T^{\prime} \in \mathcal{T},\right| T^{\prime} \cap\{u, v\} \mid=1\right\} .
\end{aligned}
$$

By the assumption we have $\alpha_{1}>0$. Also, since $u, v \in S_{x}^{i_{0}}$, we have $v \in \operatorname{dep}(x, u)$ and $u \in \operatorname{dep}(x, v)$ (by the definition of $S_{x}^{i_{0}}$ ), so that $\alpha_{2}>0$. Then, for a real number $\alpha$ such that $0<\alpha<\min \left\{\alpha_{1}, \alpha_{2}\right\}$, put $x(u) \leftarrow x(u) \pm \alpha$ and $x(v) \leftarrow x(v) \mp \alpha$ and modify $x(z)$ for $z \in U(u) \cup U(v)$ according to (B) in Lemma 3.11. (The modification of $x(w)$ for $w \in(U(u) \backslash\{u\}) \cup(U(v) \backslash\{v\})$ according to (B) can be made because the relevant components are stars. This includes the case where the relevant component is an isolated vertex in case (B2)(2). Also see the proof of the only-if part of Lemma 3.14.) Let $x^{+}$and $x^{-}$be the obtained new points. Since $\alpha_{2} \leq \min \{\tilde{c}(x, u, v), \tilde{c}(x, v, u)\}$ and since $x^{ \pm}$satisfy the assumption of Lemma 3.11 because of the choice of $\alpha$, we have $x^{ \pm} \in \mathrm{P}_{2}(f)$ and $x=\frac{1}{2}\left(x^{+}+x^{-}\right)$. This completes the proof of this lemma.

We now show the following theorem.

THEOREM 3.16. For a given $x \in \mathrm{P}_{2}(f), x$ is an extreme point of $\mathrm{P}_{2}(f)$ if and only if there exists a $T \in \mathcal{T}$ such that the following (a)-(e) hold: 
(a) For every tight set $T^{\prime} \in \mathcal{T}(x)$, we have $T^{\prime} \subseteq \bigcup\{U(u) \mid u \in T\}$.

(b) $x^{T} \in \mathrm{B}\left(f^{T}\right)$.

(c) For each $u \in T$,

(c1) if $x(u) \geq 0$, then $x(v)=-x(u)$ for all $v \in U(u) \backslash\{u\}$;

(c2) otherwise,

(1) $x(v)=x(u)$ for all $v \in U(u) \backslash\{u\}$ but one $v^{\prime}$ with $x\left(v^{\prime}\right)=-x(u)$ or

(2) $x(v)=0$ for all $v \in U(u) \backslash\{u\}$.

(d) For some covering set $W=\left\{w^{i} \mid i \in I\right\}$ of $G_{x}^{T}$ with strongly connected components having vertex sets $S_{x}^{i} \subseteq T(i \in I)$ we have $x(v)=0$ for all $v \in T \backslash W$. Moreover, for each $i \in I$ and $v \in S_{x}^{i} \backslash\left\{w^{i}\right\}$ we have $|U(v)| \geq 3$, and if values of $x(v)$ are determined by (2) of $(\mathrm{c} 2)$, we have $\left|U\left(w^{i}\right)\right| \geq 4$.

(e) For all $v \in U \in \mathcal{U}$ with $U \cap T=\emptyset$ we have $x(v)=0$. Moreover, $|U| \geq 3$ for all $U \in \mathcal{U}$ such that $U \cap T=\emptyset$.

Here conditions (b), (c), and (d) are void if $T=\emptyset$.

Proof. If (a)-(e) are satisfied for $x \in \mathrm{P}_{2}(f)$, then we have tight equations given as follows:

(3.24) $x\left(T_{i}\right)=f\left(T_{i}\right)$ for a maximal chain (3.23) of tight sets $T_{i}$ for $x^{T}$,

$(3.25) x(u)+x(v)=0 \quad(\forall u \in T, \forall v \in U(u) \backslash\{u\}$ in case $(\mathrm{c} 1))$,

(3.26) $x(v)+x(z)=0 \quad\left(\forall u \in T, \forall\{v, z\} \in\left(\begin{array}{c}U(u) \\ 2\end{array}\right)\right.$ in case (c1) with $\left.x(u)=0\right)$,

(3.27) $x\left(v^{\prime}\right)+x(v)=0 \quad\left(\forall u \in T, \forall v \in U(u) \backslash\left\{v^{\prime}\right\}\right.$ in case $\left.(\mathrm{c} 2)(1)\right)$,

(3.28) $x(v)+x(z)=0 \quad\left(\forall i \in I, \forall\{v, z\} \in\left(\begin{array}{c}U\left(w^{i}\right) \backslash\left\{w^{i}\right\} \\ 2\end{array}\right)\right.$ in case (c2)(2)),

(3.29) $x(v)+x(z)=0 \quad\left(\forall U \in \mathcal{U}\right.$ with $\left.U \cap T=\emptyset, \forall\{v, z\} \in\left(\begin{array}{l}U \\ 2\end{array}\right)\right)$.

We can see that the system of equations (3.24)-(3.29) uniquely determines the solution $x$, due to Lemma 3.14, so that $x$ is an extreme point of $\mathrm{P}_{2}(f)$.

Conversely, suppose that $x \in \mathrm{P}_{2}(f)$ is an extreme point. Then for each $u \in V$ there must exist a tight equation of type (I) or (II):

(I) $x(T)=f(T)$ for some $T \in \mathcal{T}$ with $u \in T$,

(II) $x(X)=0$ for some $X \in\left(\begin{array}{c}U \\ 2\end{array}\right)$ with $u \in X$ and $U \in \mathcal{U}$.

Denote by $\mathcal{T}(x)$ the collection of tight sets $T$ of type (I) (as before) and define $W=$ $\bigcup\{T \mid T \in \mathcal{T}(x)\}$.

Since $x \in \mathrm{P}_{2}(f)$, we have $\operatorname{dep}(x, u) \in \mathcal{T}(x)$ for all $u \in W$. Moreover, for any $u \in$ $W$ and any $v \in W \backslash \bigcup\{U \in \mathcal{U} \mid U \cap \operatorname{dep}(x, u) \neq \emptyset\}$ we have $\operatorname{dep}(x, u) \cup \operatorname{dep}(x, v) \in \mathcal{T}(x)$. Hence, similarly as in the constructive proof of Corollary 3.1, there exists $T \in \mathcal{T}(x)$ such that $T \cap U(u) \neq \emptyset$ for all $u \in W$. Let us show that for such $T$, conditions (a)-(e) are satisfied.

First, (a), (b), and (e) follow from the choice of $T$ and Lemma 3.14.

Second, we show (c). Fixing the values of $x(u)$ for all $u \in T$ and discarding the constraints $x\left(T^{\prime}\right) \leq f\left(T^{\prime}\right)$ for all $T^{\prime} \in \mathcal{T} \backslash 2^{T}$, componentwise maximal vectors $x$ satisfying (3.20) are exactly those determined by (c), due to Lemmas 3.13 and 3.14 . Hence, if $x$ does not satisfy (c), then defining $Z=\bigcup\{U(v) \mid v \in T\}$, there exist $u \in T$ and $y \in \mathbb{R}^{Z}$, defined appropriately as in Lemma 3.12, such that (i) $x^{Z} \leq y$ and (ii) $x(\hat{w})<y(\hat{w})$ for $\hat{w}$ with $\{\hat{w}\}=U(u) \cap T^{\prime}$ for a tight set $T^{\prime} \in \mathcal{T}(x)$. Since all the tight sets $T^{\prime \prime} \in \mathcal{T}(x)$ for $x$ are included in $Z$ and we have $x \in \mathrm{P}_{2}(f)$ and $y \in \mathrm{P}_{2}\left(f^{Z}\right)$ because of Lemma 3.12, defining $y^{*} \in \mathbb{R}^{V}$ by $y^{*}(v)=y(v)$ for all $v \in Z$ and $y^{*}(v)=0$ 


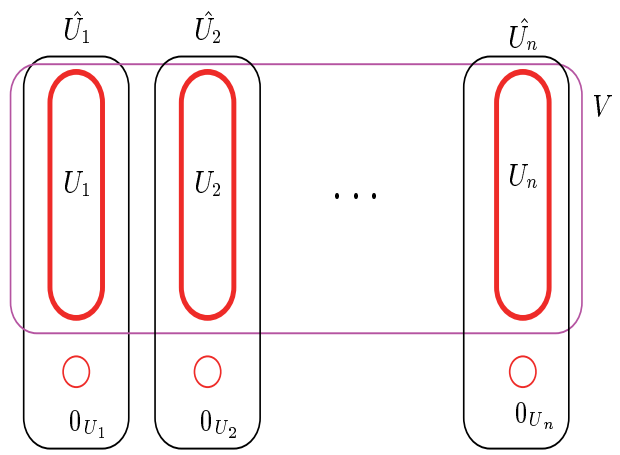

FIG. 2. An illustration of $U$ and $\hat{U}$ for $U \in \mathcal{U}$.

for all $V \backslash Z$, we have for a sufficiently small positive $\epsilon>0$

$$
z_{\epsilon} \equiv \epsilon x+(1-\epsilon) y^{*} \in \mathrm{P}_{2}(f) \text {. }
$$

Then we have $z_{\epsilon}(\hat{w})>x(\hat{w})$, which implies $z_{\epsilon}\left(T^{\prime}\right)>f\left(T^{\prime}\right)$, a contradiction. Hence (c) is satisfied.

Finally, (d) follows from Lemma 3.15 since for each $i \in I$ there exists at most one $u \in S_{x}^{i}$ such that $x(u) \neq 0$.

In the counterexample given above, $T$ appearing in Theorem 3.16 is $T=\left\{v_{1}, u_{1}\right\}$, the graph $G_{x^{*}}^{T}$ is strongly connected, and a covering set is $W=\left\{v_{1}\right\}$.

It should be noted that we have assumed the membership $x \in \mathrm{P}_{2}(f)$ in the characterization of extreme points, so that it is not well characterized so as to obtain extreme points efficiently.

4. Submodular functions on lattices. As another example of t-submodular functions we consider submodular functions on lattices and, in particular, diamonds.

Let $V, \mathcal{U}$, and $\mathcal{T}$ be those appearing in section 2 .

4.1. Min-max theorems. For each $U \in \mathcal{U}$ let $0_{U}$ be a new element and put $\hat{U}=U \cup\left\{0_{U}\right\}$. (See Figure 2.) Suppose that for each $U \in \mathcal{U}$ we are given an arbitrary lattice $\mathcal{L}_{\hat{U}}=\left(\hat{U}, \vee_{\hat{U}}, \wedge_{\hat{U}}\right)$ with lattice operations, join $\vee_{\hat{U}}$, and meet $\wedge_{\hat{U}}$, where $0_{U}$ is the minimum element of $\mathcal{L}_{\hat{U}}$. Denote by $1_{U}$ the maximum element of $\mathcal{L}_{\hat{U}}$, which belongs to $U$.

Let $\mathcal{L}=\otimes_{U \in \mathcal{U}} \mathcal{L}_{\hat{U}}\left(=\left(\otimes_{U \in \mathcal{U}} \hat{U}, \vee, \wedge\right)\right)$ be the product of lattices $\mathcal{L}_{\hat{U}}=\left(\hat{U}, \vee_{\hat{U}}, \wedge_{\hat{U}}\right)$ for $U \in \mathcal{U}$. A function $f: \otimes_{U \in \mathcal{U}} \hat{U} \rightarrow \mathbb{R}$ is called a submodular function on $\mathcal{L}$ if

$$
f(\hat{T})+f\left(\hat{T}^{\prime}\right) \geq f\left(\hat{T} \vee \hat{T}^{\prime}\right)+f\left(\hat{T} \wedge \hat{T}^{\prime}\right)
$$

for all $\hat{T}, \hat{T}^{\prime} \in \otimes_{U \in \mathcal{U}} \hat{U}$. We assume that $f\left(0_{\mathcal{L}}\right)=0$, where $0_{\mathcal{L}}$ is the minimum element of $\mathcal{L}$.

This function can be seen as a special case of t-submodular functions as follows. Note that every subtransversal $T \in \mathcal{T}$ is identified with the unique $\hat{T} \in \otimes_{U \in \mathcal{U}} \hat{U}$ satisfying

$$
\hat{T} \cap \hat{U}=\left\{\begin{array}{ll}
T \cap U & \text { if } T \cap U \neq \emptyset \\
\left\{0_{U}\right\} & \text { if } T \cap U=\emptyset
\end{array} \quad(\forall U \in \mathcal{U})\right.
$$


where $\hat{T} \in \otimes_{U \in \mathcal{U}} \hat{U}$ is regarded as a transversal of $\hat{\mathcal{U}} \equiv\{\hat{U} \mid U \in \mathcal{U}\}$. Every $T \in \mathcal{T}$ is obtained from such a transversal $\hat{T}$ of $\hat{\mathcal{U}}$ by discarding all the minimum elements $0_{U} \in \hat{T}$.

Also define for all $T, T^{\prime} \in \mathcal{T}$

$$
T \vee_{0} T^{\prime}=\left(\hat{T} \vee \hat{T}^{\prime}\right) \cap V, \quad T \wedge_{0} T^{\prime}=\left(\hat{T} \wedge \hat{T}^{\prime}\right) \cap V,
$$

where $\hat{T} \vee \hat{T}^{\prime}, \hat{T} \wedge \hat{T}^{\prime} \in \otimes_{U \in \mathcal{U}} \hat{U}$ are regarded as transversals of $\hat{\mathcal{U}}$.

For a submodular function $f$ on $\mathcal{L}$ we can identify $f$ with a function $\bar{f}$ on $\mathcal{T}$ defined by

$$
\bar{f}(T)=f(\hat{T}) \quad(\forall T \in \mathcal{T}) .
$$

Hence, we have function $\bar{f}$ with $\bar{f}(\emptyset)=0$ satisfying

$$
\bar{f}(T)+\bar{f}\left(T^{\prime}\right) \geq \bar{f}\left(T \vee_{0} T^{\prime}\right)+\bar{f}\left(T \wedge_{0} T^{\prime}\right) \quad\left(\forall T, T^{\prime} \in \mathcal{T}\right) .
$$

We can easily see that $\bar{f}$ is a t-submodular function with respect to binary operations $\vee_{0}$ and $\wedge_{0}$ (i.e., (2.2) and (2.3) are satisfied for $\nabla=\vee_{0}$ and $\triangle=\wedge_{0}$ ).

Define

$$
\mathrm{P}(\bar{f})=\left\{x \in \mathbb{R}^{V} \mid \forall T \in \mathcal{T}: x(T) \leq \bar{f}(T)\right\} .
$$

As a corollary of Theorem 2.5 we obtain the following corollary.

Corollary 4.1. For any submodular function $f$ on the product of lattices with $\bar{f}(\emptyset)=0$,

$$
\min \{\bar{f}(T) \mid T \in \mathcal{T}\}=\max \left\{-\|x\|_{1, \infty} \mid x \in \mathrm{P}(\bar{f})\right\} .
$$

Moreover, if $\bar{f}$ is integer-valued, then there exists an integral $x$ that attains the maximum on the right-hand side of (4.6).

It should be noted that because of the proof of Theorem 2.5 there exists a maximizer $x$ of the right-hand side of (4.6) such that $x$ is a $\mathcal{U}$-componentwise constant vector (i.e., $x(u)=x(v)$ for all $u, v \in U$ and all $U \in \mathcal{U}$ ) and that such a maximizer $x$ can be integral if $\bar{f}$ is integer-valued.

Motivated by a result by Kuivinen [11] (which will be examined in the next subsection), we consider the following additional constraint:

$\left(\mathrm{K} 1^{\prime}\right)$ For each $U \in \mathcal{U}, x(u)+x(v) \leq x\left(u \vee_{\hat{U}} v\right)+x\left(u \wedge_{\hat{U}} v\right)$ for all $\{u, v\} \in\left(\begin{array}{c}\hat{U} \\ 2\end{array}\right)$, where $x\left(0_{U}\right)=0$.

We define an associated polyhedron $\mathrm{P}^{\prime}(\bar{f})$ by

$$
\mathrm{P}^{\prime}(\bar{f})=\left\{x \mid x \in \mathrm{P}(\bar{f}),\left(\mathrm{K} 1^{\prime}\right)\right\} .
$$

Since the $\mathcal{U}$-componentwise constant and nonpositive maximizer $x$ to be used in the proof of Corollary 4.1 as a specialization of the proof of Theorem 2.5 satisfies (K1'), we also get the following corollary.

COROLlary 4.2. For any submodular function $f$ on the product of lattices with $\bar{f}(\emptyset)=0$,

$$
\min \{\bar{f}(T) \mid T \in \mathcal{T}\}=\max \left\{-\|x\|_{1, \infty} \mid x \in \mathrm{P}^{\prime}(\bar{f})\right\} .
$$

Moreover, if $\bar{f}$ is integer-valued, then there exists an integral $x$ that attains the maximum on the right-hand side of (4.8). 


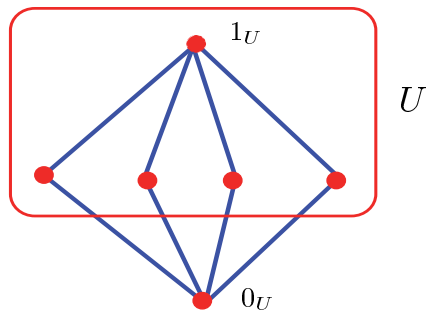

Fig. 3. A diamond.

For any $x \in \mathrm{P}(\bar{f})$ we call $T \in \mathcal{T}$ x-tight if $x(T)=\bar{f}(T)$. The following lemma will frequently be used later.

Lemma 4.3. Suppose we are given a vector $x \in \mathbb{R}^{V}$ satisfying $\left(\mathrm{K} 1^{\prime}\right)$. Then, $x$ regarded as a function on $\mathcal{T}$ is supermodular, i.e., $x(T)+x\left(T^{\prime}\right) \leq x\left(T \vee_{0} T^{\prime}\right)+x\left(T \wedge_{0} T^{\prime}\right)$ for all $T, T^{\prime} \in \mathcal{T}$.

Moreover, if $x \in \mathrm{P}^{\prime}(\bar{f})$ and $T, T^{\prime} \in \mathcal{T}$ are $x$-tight, then $T \vee_{0} T^{\prime}$ and $T \wedge_{0} T^{\prime}$ are also $x$-tight, and for each $U \in \mathcal{U}, x(u)+x(v)=x\left(u \vee_{\hat{U}} v\right)+x\left(u \wedge_{\hat{U}} v\right)$ holds for $u \in \hat{T} \cap \hat{U}$ and $v \in \hat{T}^{\prime} \cap \hat{U}$, where $x\left(0_{U}\right)=0$.

Proof. Since $x$ satisfies $\left(\mathrm{K} 1^{\prime}\right), x$ is supermodular on $\mathcal{T}$. It then follows that $\bar{f}-x$ is submodular and nonnegative when $x \in \mathrm{P}^{\prime}(\bar{f})$. Hence, the latter part of this lemma holds, where modularity follows from submodularity of $\bar{f}$ and supermodularity of $x$.

4.2. Submodular functions on diamonds. Corollary 4.2 looks like a straightforward consequence of Theorem 2.5, but it turns out that it already gives a good characterization in the case when each $\mathcal{L}_{\hat{U}}$ is a diamond. Before showing it (in section 4.2.2) let us first examine its connection with a result by Kuivinen [11].

4.2.1. Kuivinen's min-max theorem. We assume that $|U| \geq 3$ and all the elements in $U \backslash\left\{1_{U}\right\}$ are incomparable in $\mathcal{L}_{\hat{U}}$ for each $U \in \mathcal{U}$. Then the lattice $\mathcal{L}_{\hat{U}}$ on $\hat{U}=U \cup\left\{0_{U}\right\}$ is called a diamond. (See Figure 3.) We assume that for each $U \in \mathcal{U}$ $\mathcal{L}_{\hat{U}}$ is a diamond.

Corollary 4.2 gives a min-max formula for a submodular function on the product lattice of diamonds. Note that in this special case $\left(\mathrm{K}^{\prime}\right)$ is simplified to the following:

$\left(\mathrm{K}^{\prime}\right)$ For each $U \in \mathcal{U}, x(u)+x(v) \leq x\left(1_{U}\right)$ for all $\{u, v\} \in\left(\begin{array}{c}\bar{U} \\ 2\end{array}\right)$, where $\bar{U}=U \backslash\left\{1_{U}\right\}$. Kuivinen [11] considered the following stronger constraints:

(K1) For each $U \in \mathcal{U} \quad x\left(1_{U}\right)=\max \left\{x(u)+x(v) \mid\{u, v\} \in\left(\begin{array}{c}\bar{U} \\ 2\end{array}\right)\right\}$.

(K2) For each $U \in \mathcal{U}$ there exists $p \in \bar{U}$ such that $x(p) \geq x(v)$ for all $v \in \bar{U}$ and $x(u)=x(v)$ for all $u, v \in \bar{U} \backslash\{p\}$. (Such an $x$ is called unified in [11].)

Note that (K1) implies $\left(\mathrm{K} 1^{\prime}\right)$.

Kuivinen [11] showed the following theorem. We will prove it by using Corollary 4.2 and the property (being $\mathcal{U}$-componentwise constant and nonpositive) of a maximizer appearing in the proof of Theorem 2.5.

THEOREM 4.4 .

$$
\min \{\bar{f}(T) \mid T \in \mathcal{T}\}=\max \left\{\sum_{U \in \mathcal{U}} x\left(1_{U}\right) \mid x \in \mathrm{P}(\bar{f}), x \leq \mathbf{0},(\mathrm{K} 1),(\mathrm{K} 2)\right\} .
$$

Moreover, if $\bar{f}$ is integer-valued, then there exists an integral $x$ that attains the maxi- 
mum on the right-hand side of (4.9).

In order to prove this theorem we need one more term and a lemma. We call $v \in V$ saturated for $x \in \mathrm{P}(\bar{f})$ if there is an $x$-tight set that contains $v$. Lemma 4.3 is specialized to the product lattice of diamonds as follows.

Lemma 4.5. Let $\{u, v\} \in\left(\begin{array}{c}\bar{U} \\ 2\end{array}\right)$ for some $U \in \mathcal{U}$. Suppose that $u$ and $v$ are saturated for $x \in \mathrm{P}^{\prime}(\bar{f})$. Then $x(u)+x(v)=x\left(1_{U}\right)$ holds.

Proof of Theorem 4.4. We can easily see the weak duality, i.e., for any $T \in \mathcal{T}$ and any $x \in \mathrm{P}(\bar{f})$ such that $x \leq \mathbf{0}$ and (K1) and (K2) are satisfied, we have the inequality

$$
\bar{f}(T) \geq \sum_{U \in \mathcal{U}} x\left(1_{U}\right)
$$

Let $x^{*}$ be a maximizer of the right-hand side of (4.8). As we remarked before, such a maximizer $x^{*}$ can be taken as a nonpositive $\mathcal{U}$-componentwise constant vector, i.e., $x^{*} \leq \mathbf{0}$ and $x^{*}(u)=x^{*}(v)$ for $u, v \in U$ and all $U \in \mathcal{U}$.

Put $y \leftarrow x^{*}$. For each $U \in \mathcal{U}$, let us choose any element from $\bar{U}=U \backslash\left\{1_{U}\right\}$ and denote it by $p_{U}$. We increase the value of $y\left(p_{U}\right)$ as much as possible while keeping $y \leq \mathbf{0},\left(\mathrm{K}^{\prime}\right)$, and $y \in \mathrm{P}(\bar{f})$. Denote the resulting $y$ by $y^{*}$.

Note that $x^{*}\left(p_{U}\right) \leq y^{*}\left(p_{U}\right)$ and $x^{*}(v)=y^{*}(v)$ for all $v \in U \backslash\left\{p_{U}\right\}$, and hence (K2) is satisfied for $y^{*}$. We will modify $y^{*}$ so that (K1) becomes satisfied while keeping (K2), by doing the following for each $U \in \mathcal{U}$.

Initially, for each $U \in \mathcal{U}$ one of the following two holds: (i) $y^{*}\left(p_{U}\right)=0$ (or $y^{*}\left(p_{U}\right)+y^{*}(v)=y^{*}\left(1_{U}\right)$ for all $v \in U \backslash\left\{1_{U}, p_{U}\right\}$ ) and (ii) $p_{U}$ is saturated for $y^{*}$. If (i) holds, (K1) and (K2) are satisfied for $U$.

Hence, suppose that $p_{U}$ is saturated for $y^{*}$ and $y^{*}\left(p_{U}\right)<0$. Then increase the values of $y^{*}(v)$ for all $v \in U \backslash\left\{1_{U}, p_{U}\right\}$ by the same value as much as possible while keeping $y^{*}(v) \leq y^{*}\left(p_{U}\right),\left(\mathrm{K} 1^{\prime}\right),(\mathrm{K} 2)$, and $y^{*} \in \mathrm{P}(\bar{f})$. If some $v \in U \backslash\left\{1_{U}, p_{U}\right\}$ is saturated for the new $y^{*}$, we have $y^{*}\left(p_{U}\right)+y^{*}(v)=y^{*}\left(1_{U}\right)$ by Lemma 4.5 and thus $y^{*}$ satisfies (K1) and (K2) for $U$. Therefore, suppose that $y^{*}\left(p_{U}\right)+y^{*}(v)<y^{*}\left(1_{U}\right)$ for all $v \in U \backslash\left\{1_{U}, p_{U}\right\}$. Then we have $y^{*}(v)=y^{*}\left(p_{U}\right)$ for all $v \in U \backslash\left\{1_{U}, p_{U}\right\}$. We pick any element in $U \backslash\left\{1_{U}, p_{U}\right\}$ and denote it by $q_{U}$. We then increase the value of $y^{*}\left(q_{U}\right)$ as much as possible while keeping $\left(\mathrm{K}^{\prime}\right)$ and $y^{*} \in \mathrm{P}(\bar{f})$. Note that for the resulting $y^{*}$ we have $y^{*}\left(q_{U}\right) \leq 0$ since $y^{*}\left(q_{U}\right) \leq y^{*}\left(1_{U}\right)-y^{*}\left(p_{U}\right) \leq x^{*}\left(1_{U}\right)-x^{*}\left(p_{U}\right)=0$. Hence, $y^{*}\left(p_{U}\right)+y^{*}\left(q_{U}\right)=y^{*}\left(1_{U}\right)$ holds or $q_{U}$ is saturated for $y^{*}$, where the latter case also implies $y^{*}\left(p_{U}\right)+y^{*}\left(q_{U}\right)=y^{*}\left(1_{U}\right)$ by Lemma 4.5. Therefore, the resulting $y^{*}$ satisfies (K1) and (K2) for $U$.

Since $y^{*} \in \mathrm{P}^{\prime}(\bar{f}), x^{*} \leq y^{*} \leq \mathbf{0}$, and $-\left\|x^{*}\right\|_{1, \infty} \leq-\left\|y^{*}\right\|_{1, \infty} \leq \sum_{U \in \mathcal{U}} y^{*}\left(1_{U}\right)$, Theorem 4.4 follows from Corollary 4.2 and the weak duality (4.10). Here also note that when $\bar{f}$ is integer-valued, $x^{*}$ can be chosen to be integral and the integrality of $y^{*}$ is kept throughout the above argument.

4.2.2. A good characterization. Kuivinen showed that Theorem 4.4 gives a good characterization of the minimization problem of $\bar{f}$. Here we show that Corollary 4.2 also gives a good characterization in the case when $\mathcal{L}$ is the product lattice of diamonds. This implies that only $\left(\mathrm{K} 1^{\prime}\right)$ is essential.

We should remark that the proofs of the following two theorems given here are almost direct adaptations of Kuivinen's technique [11].

We define a partial order $\preceq$ on $\mathcal{T}$ in such a way that $T \preceq T^{\prime}$ if for each $U \in \mathcal{U}$ $u \preceq_{\hat{U}} v$ holds for $u, v \in \hat{U}$ such that $\hat{T} \cap \hat{U}=\{u\}$ and $\hat{T}^{\prime} \cap \hat{U}=\{v\}$, where $\preceq_{\hat{U}}$ denotes the partial order in $\mathcal{L}_{\hat{U}}$. Note that the partial order on $\mathcal{T}$ can be identified with the partial order in the product lattice $\mathcal{L}$. 
THEOREM 4.6. Let $f$ be a submodular function on the product lattice of diamonds with $\bar{f}(\emptyset)=0$. If $x \in \mathbb{R}^{V}$ is an extreme point of $\mathrm{P}^{\prime}(\bar{f})$, then there exists a chain of $x$-tight sets $\emptyset=T_{0} \prec T_{1} \prec \cdots \prec T_{k}$ satisfying

(i) for each $U \in \mathcal{U}, T_{k} \cap U \neq \emptyset$, i.e., $T_{k}$ is a transversal of $\mathcal{U}$, and

(ii) for each $i \in\{1, \ldots, k\}$, defining $\mathcal{U}_{i}=\left\{U \mid T_{i-1} \cap U \neq T_{i} \cap U\right\}$, there exists at most one $U \in \mathcal{U}_{i}$ such that $T_{i-1} \cap U=\emptyset$ and $T_{i} \cap U=\left\{1_{U}\right\}$.

Proof. Let $\mathcal{C}: \emptyset=T_{0} \prec T_{1} \prec \cdots \prec T_{k}$ be a maximal chain of $x$-tight sets. It should be noted that $T_{k}$ is the unique maximal $x$-tight set with respect to partial order $\preceq$ on $\mathcal{T}$ (where we recall that the collection $\mathcal{T}(x)$ of $x$-tight sets is closed with respect to $\vee_{0}$ due to the latter half of Lemma 4.3). We show that chain $\mathcal{C}$ satisfies (i) and (ii).

Since $x$ is an extreme point, there is a vector $c \in \mathbb{R}^{V}$ such that $x$ is a unique maximizer of LP: $\max \left\{\langle c, x\rangle \mid x \in \mathrm{P}^{\prime}(\bar{f})\right\}$. Since the LP is bounded, we may assume that for each $U \in \mathcal{U}, c(v)>0$ for all $v \in U$ and $\sum_{u \in \bar{U}} c(u)+2 c\left(1_{U}\right)>0$.

For any $\alpha \in \mathbb{R}$ and $U \in \mathcal{U}$, define $\chi_{U, \alpha}=\sum_{v \in \bar{U}} \alpha \chi_{v}+2 \alpha \chi_{1_{U}}$, where $\chi_{v}$ denotes the characteristic vector of $\{v\}$ in $\mathbb{R}^{V}$. Note that $x+\chi_{U, \alpha}$ satisfies $\left(\mathrm{K}^{\prime}\right)$ for any $U$ and $\alpha$.

Observe that for each $U \in \mathcal{U}$ there is an $x$-tight set $T_{U}$ with $T_{U} \cap U \neq \emptyset$, since otherwise, setting $x^{\prime}=x+\chi_{U, \alpha}$ for some small $\alpha>0$, we have $x^{\prime} \in \mathrm{P}^{\prime}(\bar{f})$ and $\left\langle x^{\prime}, c\right\rangle>$ $\langle x, c\rangle$, which is a contradiction. Since we have an $x$-tight set $\bigvee_{0}\left\{T_{U} \mid U \in \mathcal{U}\right\} \preceq T_{k}$, the chain $\mathcal{C}$ satisfies (i).

To prove (ii), suppose to the contrary that there exist some index $i \in\{1, \ldots, k\}$ and distinct $U_{p}, U_{q} \in \mathcal{U}$ such that $T_{i-1} \cap U_{j}=\emptyset$ and $T_{i} \cap U_{j}=\left\{1_{U_{j}}\right\}$ for $j \in$ $\{p, q\}$. Since $c$ can be chosen generically, we may assume $\sum_{u \in \bar{U}_{p}} c(u)+2 c\left(1_{U_{p}}\right)>$ $\sum_{u \in \bar{U}_{q}} c(u)+2 c\left(1_{U_{q}}\right)$ without loss of generality. Then, putting $x^{\prime}=x+\chi_{U_{p}, \alpha}-\chi_{U_{q}, \alpha}$, we have $\left\langle c, x^{\prime}\right\rangle>\langle c, x\rangle$ for any $\alpha>0$. This implies that there is an $x$-tight set $T$ for which $T \cap U_{p} \neq \emptyset$ and $T \cap U_{q} \neq\left\{1_{U_{q}}\right\}$. Then define $T^{\prime}=\left(T \wedge_{0} T_{i}\right) \vee_{0} T_{i-1}$, which is an $x$-tight set satisfying $T_{i-1} \prec T^{\prime} \prec T_{i}$. This contradicts the maximality of the chain $\mathcal{C}$. Hence, (ii) is satisfied.

THEOREM 4.7. Let $f$ be a submodular function on the product lattice of diamonds with $\bar{f}(\emptyset)=0$. Given a vector $x \in \mathbb{R}^{V}$ and a chain of sets $\emptyset=T_{0} \prec T_{1} \prec \cdots \prec T_{k}$ in $\mathcal{T}$ satisfying $x\left(T_{i}\right)=\bar{f}\left(T_{i}\right)(i=0,1, \ldots, k)$ and (i) and (ii) of Theorem 4.6, one can check whether $x \in \mathrm{P}^{\prime}(\bar{f})$ or not in polynomial time.

Proof. Define $T_{k+1}=\left\{1_{U} \mid U \in \mathcal{U}\right\}$. We first prove the following claim.

Claim. Suppose that $x$ satisfies $\left(\mathrm{K}^{\prime}\right)$. Then, $x \in \mathrm{P}^{\prime}(\bar{f})$ holds if and only if $x$ satisfies the following:

(**) for each $i=1, \ldots, k+1, \quad x(T) \leq \bar{f}(T)$ for all $T \in \mathcal{T}$ with $T_{i-1} \preceq T \preceq T_{i}$.

Proof. The "only if" part is immediate. Hence, assume that $x$ satisfies (**). We prove the following:

(†) for each $i=0,1, \ldots, k+1, x(T) \leq \bar{f}(T)$ for all $T \in \mathcal{T}$ with $T \preceq T_{i}$, by induction on $i$ from $i=0$ through $i=k+1$.

The base case when $i=0$ is clear.

Suppose that $(\dagger)$ holds for some $i \in\{0, \ldots, k\}$ and put $i \leftarrow i+1$. Let us consider any $T \in \mathcal{T}$ with $T \preceq T_{i}$. Since $T_{i-1} \preceq T \vee_{0} T_{i-1} \preceq T_{i}$ and $T \wedge_{0} T_{i-1} \preceq T_{i-1}$, we have $x\left(T \vee_{0} T_{i-1}\right) \leq \bar{f}\left(T \vee_{0} T_{i-1}\right)$ and $x\left(T \wedge_{0} T_{i-1}\right) \leq \bar{f}\left(T \wedge_{0} T_{i-1}\right)$. Therefore, because of 
the supermodularity of $x$ (due to $\left.\left(\mathrm{K}^{\prime}\right)\right)$ and the submodularity of $\bar{f}$ we get

$$
\begin{aligned}
\bar{f}(T)+\bar{f}\left(T_{i-1}\right) & \geq \bar{f}\left(T \vee_{0} T_{i-1}\right)+\bar{f}\left(T \wedge_{0} T_{i-1}\right) \\
& \geq x\left(T \vee_{0} T_{i-1}\right)+x\left(T \wedge_{0} T_{i-1}\right) \\
& \geq x(T)+x\left(T_{i-1}\right) .
\end{aligned}
$$

This implies $x(T) \leq \bar{f}(T)$ since $T_{i-1}$ is $x$-tight.

Obviously one can check $\left(\mathrm{K}^{\prime}\right)$ in polynomial time. Hence assume that $x$ satisfies $\left(\mathrm{K} 1^{\prime}\right)$. Then, in order to prove the present theorem it suffices to show that we can check whether $(* *)$ holds in polynomial time.

Suppose that $1 \leq i<k+1$. Also suppose that there is $U^{*} \in \mathcal{U}\left(T_{i}\right)$ such that $U^{*} \cap T_{i-1}=\emptyset$ and $U^{*} \cap T_{i}=\left\{1_{U^{*}}\right\}$, where recall the notation (2.1). Define

$$
T^{*}=\left\{v \in V \mid U \in \mathcal{U}, T_{i-1} \cap U \neq T_{i} \cap U, T_{i} \cap U=\{v\}\right\} \backslash U^{*} .
$$

It follows from (ii) of Theorem 4.6 that for each $v \in U^{*}$ the set, denoted by $\mathcal{T}_{v}^{*}$, of all $T \in \mathcal{T}$ with $T_{i-1} \preceq T \preceq T_{i}$ and $T \cap U^{*}=\{v\}$ is equal to $\left\{\left(T_{i-1} \vee_{0} S\right) \cup\{v\} \mid S \subseteq T^{*}\right\}$. Hence, for each $v \in U^{*}$ we can regard the function $\bar{f}$ restricted to $\mathcal{T}_{v}^{*}$ as an ordinary submodular (set) function on $2^{T^{*}}$. Consequently, by using any existing submodular function minimization algorithm, we can check whether $x(T) \leq \bar{f}(T)$ for all $T \in \mathcal{T}$ with $T_{i-1} \preceq T \preceq T_{i}$ in polynomial time.

If there is no $U^{*} \in \mathcal{U}\left(T_{i}\right)$ such that $U^{*} \cap T_{i-1}=\emptyset$ and $U^{*} \cap T_{i}=\left\{1_{U^{*}}\right\}$, then the set $\mathcal{T}^{*}$ of all $T \in \mathcal{T}$ with $T_{i-1} \preceq T \preceq T_{i}$ is equal to $\left\{T_{i-1} \vee_{0} S \mid S \subseteq T^{*}\right\}$, and the function $\bar{f}$ restricted to $\mathcal{T}^{*}$ can be regarded as an ordinary submodular function on $2^{T^{*}}$. Thus we can check whether $x(T) \leq \bar{f}(T)$ for all $T \in \mathcal{T}$ with $T_{i-1} \preceq T \preceq T_{i}$ in polynomial time.

Finally, if $i=k+1$, then condition (i) of Theorem 4.6 implies that there is no $U^{*} \in \mathcal{U}$ such that $U^{*} \cap T_{k}=\emptyset$. Therefore, the same argument can be applied again to check whether $x(T) \leq \bar{f}(T)$ for all $T \in \mathcal{T}$ with $T_{k} \preceq T\left(\preceq T_{k+1}\right)$ in polynomial time.

Theorems 4.6 and 4.7 imply that Corollary 4.2 is a good characterization of the minimization problem of $f$. Indeed, suppose that one wants to decide whether $T \in$ $\mathcal{T}$ is a minimizer of $\bar{f}$. Then, if the answer is no, there is a certificate $T^{\prime}$ with $\bar{f}\left(T^{\prime}\right)<\bar{f}(T)$. On the other hand, if the answer is yes, then from Theorem 4.6 (and fundamental facts on polyhedra) there is a certificate which consists of $y \in \mathbb{R}^{V}, z_{i} \in$ $\mathbb{R}^{V}(i=1, \ldots, m), T_{0}^{i}, T_{1}^{i}, \ldots, T_{k_{i}}^{i} \in \mathcal{T}(i=1, \ldots, m)$, and $\lambda_{i} \in(0,1](i=1, \ldots, m)$ for some $m$ with $1 \leq m \leq|V|+|\mathcal{U}|$ such that

- $y$ is a point in the characteristic cone of $\mathrm{P}^{\prime}(\bar{f})$;

- $\sum_{i=1}^{m} \lambda_{i}=1$;

- $\forall i=1, \ldots, m: \emptyset=T_{0}^{i} \prec T_{1}^{i} \prec \cdots \prec T_{k_{i}}^{i}$ and $z_{i}\left(T_{j}^{i}\right)=\bar{f}\left(T_{j}^{i}\right)\left(\forall j=1, \ldots, k_{i}\right)$;

- $\forall i=1, \ldots, m: z_{i}$ and $T_{0}^{i} \prec \cdots \prec T_{k_{i}}^{i}$ satisfy conditions (i) and (ii) of Theorem 4.6 ;

- $\forall i=1, \ldots, m: z_{i} \in \mathrm{P}^{\prime}(\bar{f})$;

- $-\|x\|_{1, \infty}=\hat{f}(T)$, where $x=y+\sum_{i=1}^{m} \lambda_{i} z_{i}$.

Since these conditions can be checked in polynomial time by Theorem 4.7 , we can conclude that $T$ is a minimizer of $\bar{f}$ by Corollary 4.2.

In case of $k$-submodular functions, condition (ii) of Theorem 4.6 may not hold when adopting the same approach, which would become an obstacle to get a good characterization for the minimization of $k$-submodular functions. 
5. Concluding remarks. We have shown a min-max theorem for t-submodular functions in terms of a new norm composed of $\ell_{1}$ and $\ell_{\infty}$ norms, which reveals its relation to the min-max theorem for ordinary submodular set functions due to Edmonds [4]. The obtained min-max relation looks nice but it is not clear whether the min-max relation gives us a good characterization in general. In particular, a good characterization for minimizing $k$-submodular functions is still open. In order to get a good characterization we have some degrees of freedom in choosing polyhedra appearing on the side of maximization. Finding a right polyhedron that leads us to a good characterization is left for future research.

Devising a polynomial-time algorithm for minimizing $k$-submodular functions in the value oracle model is also left open, where note that such a polynomial-time algorithm would provide us with a good characterization for minimizing $k$-submodular functions. As pointed out in [7] and discussed here as well, we need a good characterization of extreme points of $\mathrm{P}_{2}(f)$. A key to the good characterization is to develop a polynomial-time algorithm for linear optimization over $\mathrm{P}_{2}(f)$. The main difficulty in linear optimization over $\mathrm{P}_{2}(f)$ is that a polynomial-time algorithm for it requires an efficient membership algorithm for discerning whether $\mathbf{0} \in \mathrm{P}(f)$.

We have also shown a min-max relation for submodular functions on product lattices of general lattices. When each component lattice is a diamond, it gives a good characterization, whose proof technique can also be adapted to show that our min-max relation gives a good characterization when each component lattice is a pentagon. It was shown by Krokhin and Larose [9] that submodular functions on pentagons can be minimized in polynomial time in the value oracle model, while the tractability on diamonds is still open [11], where only a pseudopolynomial algorithm is given in [11]. It is an interesting open problem to find nontrivial lattices other than diamonds and those considered in [9] for which we have a good characterization, while Kuivinen showed in his dissertation [10] that modular lattices have a good characterization.

Finally, we remark that t-submodular functions are far more general than $k$ submodular functions and submodular functions on product lattices. It is worth investigating a proper subclass of $\mathrm{t}$-submodular functions with symmetric binary operations $\nabla$ and $\triangle$ satisfying, besides (2.2) and (2.3), the condition that for all $T_{1}, T_{2} \in \mathcal{T}$, if $T_{1} \cup T_{2} \in \mathcal{T}$, then $T_{1} \nabla T_{2}=T_{1} \cup T_{2}$ and $T_{1} \triangle T_{2}=T_{1} \cap T_{2}$. Note that $k$-submodular functions and submodular functions on product lattices belong to this class.

Acknowledgments. We are grateful to the two anonymous referees for their careful reading of and useful comments on an original version of this paper.

\section{REFERENCES}

[1] A. Bouchet, Greedy algorithm and symmetric matroids, Math. Programming, 38 (1987), pp. $147-159$.

[2] A. Bouchet, Multimatroids I-Coverings by independent sets, SIAM J. Discrete Math., 10 (1997), pp. 626-646.

[3] A. Bouchet and W. H. Cunningham, Delta-matroids, jump systems, and bisubmodular polyhedra, SIAM J. Discrete Math., 8 (1995), pp. 17-32.

[4] J. Edmonds, Submodular functions, matroids, and certain polyhedra, in Proceedings of the Calgary International Conference on Combinatorial Structures and Their Applications, R. Guy, H. Hanani, N. Sauer, and J. Schönheim, eds., Gordon and Breach, New York, 1970, pp. 69-87.

[5] S. Fujishige, A min-max theorem for bisubmodular polyhedra, SIAM J. Discrete Math., 10 (1997), pp. 294-308. 
[6] S. Fujishige, Submodular Functions and Optimization, 2nd ed., Ann. Discrete Math. 58, Elsevier, Amsterdam, 2005.

[7] A. Huber and V. Kolmogorov, Towards minimizing $k$-submodular functions, in Proceedings of ISCO 2012, Lecture Notes in Comput. Sci. 7422, Springer, Heidelberg, 2012, pp. 451-462.

[8] V. Kolmogorov, Submodularity on a tree: Unifying $L^{\natural}$-convex and bisubmodular functions, in Proceedings of MFCS 2011, Lecture Notes in Comput. Sci. 6907, Springer, Heidelberg, 2011, pp. 400-411.

[9] A. Krokhin And B. Larose, Maximizing supermodular functions on product lattices, with application to maximum constraint satisfaction, SIAM J. Discrete Math., 22 (2008), pp. 312-328.

[10] F. Kuivinen, Algorithms and Hardness Results for Some Valued CSPs, Dissertation No. 1274, Linköping Studies in Science and Technology, Linköping University, Linköping Sweden, 2009.

[11] F. Kuivinen, On the complexity of submodular function minimisation on diamonds, Discrete Optim., 8 (2011), pp. 459-477.

[12] J. ThAPPER AND S. ŽIVNÝ, The power of linear programming for valued CSPs, in Proceedings of FOCS 2012, IEEE, Piscataway, NJ, 2012, pp. 669-678.

[13] J. Thapper and S. ŽIVnÝ, The complexity of finite valued CSPs, in Proceedings of the Fortyfifth Annual ACM Symposium on Theory of Computing 2013, ACM, New York, pp. 695704.

[14] S. ŽIvnÝ, The Complexity of Valued Constraint Satisfaction Problems, Springer, Heidelberg, 2012 